\title{
ALTERAÇÕES NA MICROBIOLOGIA DO SOLO E CULTIVO DE ALFACE (LACTUCA SATIVA L.), EM FUNÇÃO DA COBERTURA DO SOLO COM PALHADAS.
}

Heloisa Maria da Cruz Jardim; Fabio Fernando de Araujo

Universidade do Oeste Paulista - UNOESTE, Curso de Agronomia, Presidente Prudente, SP. E-mail: heloisa.jardim@yahoo.com.br

\section{RESUMO}

O objetivo do trabalho foi avaliar o efeito de cobertura com palhadas sobre a microbiologia do solo refletindo no aumento do crescimento de plantas olerícolas. O experimento foi realizado em Álvares Machado no sitio São Lourenço. Foram avaliados dois tipos de resíduos como Casca de Amendoim e Cana de Açúcar triturada e também dois níveis de cobertura do solo de 3 e $6 \mathrm{~cm}$ em cultivos sucessivos. O estudo foi conduzido em canteiros de horta comercial, atendendo ao manejo de condução das plantas adotado pelos produtores. Trinta dias após o plantio foram coletada amostra de solo para análise microbiológica, avaliaram-se as plantas invasoras. A utilização da cobertura morta trouxe resultados benéficos ao solo, como à redução de ervas espontânea, o uso da casca de amendoim melhorou a biomassa microbiana do solo e aumentou a produtividade da alface nos dois cultivos, sobressaindo o nível de $3 \mathrm{~cm}$.

Palavras-chave: Atividade microbiana, resíduos orgânicos, horticultura, produtividade, alface.

\section{ALTERATIONS IN THE SOIL MICROBIOLOGY AND CACTIVE OF LETTUCE (LACTUCA SATIVA L.), IN THE FUNCTION OF THE COVERAGE OF THE SOIL WITH STRAWS}

\begin{abstract}
The objective of this work was to evaluate the effect of mulch coverage on soil microbiology, reflecting the growth of olerocarpa plants. The experiment was carried out in Álvares Machado at the São Lourenço site. Two types of residues such as Peanut Peel and Crushed Sugarcane were evaluated, as well as two levels of soil cover of 3 and $6 \mathrm{~cm}$ in successive crops. The study was conducted in commercial vegetable garden beds, taking into account the management of the plants adopted by the producers. Thirty days after planting, soil samples were collected for microbiological analysis, and invasive plants were evaluated. The use of mulch resulted in beneficial soil effects, such as the reduction of spontaneous grasses, the use of peanut bark improved soil microbial biomass and increased lettuce productivity in both crops, with a $3 \mathrm{~cm}$ level.
\end{abstract}

Keywords: Microbial activity, organic waste, horticulture, productivity, lettuce.

\section{INTRODUÇÃO}

O Estado de São Paulo possui o maior setor produtivo de olerícolas no Brasil, com 23,0\% da produção e o principal mercado consumidor (22,0\% da população) (CAMARGO FILHO, 2013). A cobertura morta do solo é uma prática cultural que traz reconhecidos benefícios aos sistemas de produção, especialmente no que diz respeito à olericultura (OLIVEIRA et al, 2008). Tanto a cobertura com plástico quanto com restos vegetais têm sido exploradas com os objetivos de reduzir a evaporação da água na superfície do solo; diminuir as oscilações de temperatura do solo (ARAÚJO et al., 1993; ANDRADE JÚNIOR et al., 2005); permitir o controle de plantas invasoras; oferecer proteção aos frutos, evitando seu contato direto com o solo; obter maior precocidade da 
colheita e capacidade de influir diretamente, de maneira positiva, sobre a incidência de pragas e doenças (CASTELLANE, 1995; ANDRADE JÚNIOR et al., 2005). Segundo Müller (1991) e Resende et al. (2005), a cobertura do solo em hortaliças tem sido utilizada com o intuito de reduzir a desagregação do solo.

O solo é um sistema heterogêneo, descontínuo e estruturado, formado por microhabitats com diferentes características químicas, físicas e biológicas altamente interdependentes, de modo que o ambiente solo pode ser alterado em consequência do desmatamento, da remoção de sua camada superficial e intenso revolvimento, com impacto negativo imediato na sua microbiota e em seus processos (VALARINI et al., 2011). Sabe-se que quanto maior a quantidade de material orgânico, maior será a biomassa microbiana do solo (BMS). E também, representa uma reserva considerável de nutrientes, os quais são continuamente assimilados durante os ciclos de crescimento dos diferentes organismos que compõem o ecossistema (ARAÚJO; MONTEIRO, 2007; BATISTA et al., 2013).

Com isso o presente trabalho apresentou objetivo de avaliar o efeito de cobertura com palhadas sobre a microbiologia do solo, plantas invasoras e também sobre o crescimento e produção de Alface.

\section{METODOLOGIA}

O experimento foi realizado no período de dezembro de 2016 a maio de 2017, em horta comercial localizado no município de Álvares Machado, São Paulo, no sítio São Lourenço. O clima da região é considerado subtropical tendo uma temperatura média de 23 으 e uma precipitação de $1244 \mathrm{~mm}$, onde o verão é bem quente e inverno com temperatura moderada e pouca chuva.

O local de desenvolvimento do experimento já era cultivado com hortaliças em canteiros por vários anos. Neste local foram reservados canteiros para realização do experimento. Foram utilizados dois tipos de resíduos no experimento: casca de amendoim e bagaço de cana. Os resíduos foram dispostos nos canteiros visando formação de dois níveis de altura do resíduo em relação ao solo: 3 e $6 \mathrm{~cm}$. Desta forma o delineamento experimental ficou definido como um fatorial $2 \times 2+1$, com quatro repetições. Onde o tratamento +1 será o cultivo no solo sem nenhuma cobertura. As parcelas (resíduos) e subparcelas (níveis) foram demarcadas dentro dos canteiros com 20 metros de comprimento por um metro de largura. Nesses canteiros foram definidas as parcelas de 10 metros de comprimentos e as subparcelas de 5 metros de comprimento. Com a adubação de plantio foi de $500 \mathrm{~kg} \mathrm{ha}^{-1}$.

Cultivou-se a alface (Lactuca sativa), cultivar Vanda. A produção das mudas, transplantes e condução da cultura foram realizadas de acordo com as recomendações técnicas regionais. A área experimental era utilizada a irrigação por aspersão convencional.

A avaliação das plantas daninhas foi realizada 20 dias após o plantio. Decorridos 30 dias de cultivo do segundo ciclo, foram coletadas amostras de solo para realização de análises microbiana seguindo a metodologia de Reichardt, (1988). Após o período de cultivo foram analisados os parâmetros de crescimento da cultura pela avaliação de componentes de produção como número de folhas, massa comercial fresca da planta, diâmetro do caule e diâmetro da parte aérea. 0 experimento se repetiu durante dois ciclos de cultivo.

Para análise estatística utilizou-se o programa Assistat. Para análise de variância foi utilizado o teste de Dunnet e Tukey a 5\% de probabilidade.

\section{RESULTADOS}

Nos canteiros de alface as espécies predominantes de plantas invasoras no primeiro ciclo de cultivo foram a Digitaria horizontalis e Eleusine indica (Tabela 1). Houve diferença significativa entre os canteiros com as coberturas para com a testemunhas, as coberturas de Casca de Amendoim e Cana Triturada de $6 \mathrm{~cm}$ apresentaram melhor controle das ervas espontâneas. No 
segundo ciclo de cultivo as ervas espontâneas apresentaram um melhor controle em ambos os manejos (Tabela 2), porém o uso das coberturas mortas obteve um menor número de plantas daninhas comparado com a testemunha.

Tabela 1. Avaliação de plantas daninhas presente nos canteiros de Alface cultivada sobre diferentes resíduos e níveis de cobertura, no primeiro ciclo de cultivo. (1 cultivo)

\begin{tabular}{|c|c|c|c|c|c|c|}
\hline \multirow{3}{*}{ Espécies daninhas } & \multicolumn{2}{|c|}{ Casca de Amendoim } & \multicolumn{2}{|c|}{ Cana Triturada } & \multirow[t]{2}{*}{ Testem. } & \multirow{3}{*}{ TOTAL } \\
\hline & $3 \mathrm{~cm}$ & $6 \mathrm{~cm}$ & $3 \mathrm{~cm}$ & $6 \mathrm{~cm}$ & & \\
\hline & \multicolumn{5}{|c|}{ Plantas daninhas $\left(\mathrm{m}^{2}\right)$} & \\
\hline $\begin{array}{c}\text { Acanthospermum } \\
\text { hispidium }\end{array}$ & 0,5 & 1 & 0 & 0 & 0,5 & 2,0 \\
\hline Amaranthus sp & 7 & 0 & 3,5 & 0,5 & 60,5 & 71,5 \\
\hline Cenchrus chinatus & 1,05 & 0,1 & 0,57 & 1,05 & 0,1 & 2,9 \\
\hline Cyperus sp & 0,1 & 0,1 & 0,1 & 0,1 & 5,05 & 5,5 \\
\hline Digitaria horizontalis & 21,5 & 1,05 & 20,5 & 2,55 & 134,5 & 180,1 \\
\hline Eleusine indica & 8 & 1,07 & 10,02 & 1,05 & 522 & 542,2 \\
\hline Portulaca oleracea & 2,52 & 1,05 & 0,1 & 0,57 & 56,5 & 60,8 \\
\hline Physalis angulata & 16 & 1,05 & 0,1 & 0,1 & 0,1 & 17,4 \\
\hline Richardia brasiliensis & 5,05 & 0,1 & 2,05 & 0,575 & 18 & 25,8 \\
\hline $\begin{array}{c}\text { Commelina } \\
\text { benghalensis }\end{array}$ & 0,1 & 0,1 & 0,57 & 0,1 & 0,1 & 1,0 \\
\hline Total & 61,5 & 5 & 37 & 6 & 791 & 900,5 \\
\hline
\end{tabular}


Tabela 2. Avaliação de plantas daninhas presente nos canteiros de Alface cultivada sobre diferentes resíduos e níveis de cobertura, no segundo ciclo de cultivo ( 2 cultivo).

\begin{tabular}{|c|c|c|c|c|c|c|}
\hline \multirow{3}{*}{ Espécies daninhas } & \multicolumn{2}{|c|}{ Casca de Amendoim } & \multicolumn{2}{|c|}{ Cana triturada } & \multirow{2}{*}{ Testem. } & \multirow{3}{*}{ TOTAL } \\
\hline & $3 \mathrm{~cm}$ & $6 \mathrm{~cm}$ & $3 \mathrm{~cm}$ & $6 \mathrm{~cm}$ & & \\
\hline & \multicolumn{5}{|c|}{ Plantas daninhas $\left(\mathrm{m}^{2}\right)$} & \\
\hline $\begin{array}{c}\text { Acanthospermum } \\
\text { hispidium }\end{array}$ & 1,5 & 0 & 0 & 0 & 2 & 3,5 \\
\hline Amaranthus sp & 0,5 & 0 & 1 & 0,5 & 19,5 & 21,5 \\
\hline Brachiaria Plantaginea & 1,5 & 2,5 & 4 & 1,5 & 4,5 & 14 \\
\hline Cenchrus chinatus & 0 & 0 & 0 & 0,5 & 0 & 0,5 \\
\hline Cyperus sp & 0,5 & 1 & 0 & 0 & 1,5 & 3 \\
\hline Digitaria horizontalis & 3,5 & 3 & 10 & 4 & 41,5 & 62 \\
\hline Eleusine indica & 5 & 2 & 4,5 & 4,5 & 76 & 92 \\
\hline Portulaca oleracea & 1 & 2 & 1,5 & 1 & 35 & 40,5 \\
\hline Physalis angulata & 0,5 & 0 & 0,5 & 0 & 3,5 & 4,5 \\
\hline Richardia brasiliensis & 1 & 0 & 0,5 & 0 & 18,5 & 20 \\
\hline Total & 15 & 10,5 & 22 & 12 & 202 & 261,5 \\
\hline
\end{tabular}

De acordo com a tabela 3 a massa fresca da parte aérea e o número de folhas apresentaram melhor desenvolvimento na cobertura morta da casca de amendoim com o nível de três centímetros. No diâmetro da cabeça pode-se observar de acordo com a tabela 3, que todas as coberturas da Casca de Amendoim utilizadas possibilitaram um maior diâmetro de plantas. Enquanto que a utilizações da testemunha e Cana Triturada diminuíram o diâmetro da cabeça da alface. Nos níveis de Cana Triturada de três e seis centímetros, apresentaram um menor diâmetro de caule comparando-os com a testemunha que não se diferiram dos níveis da casca de amendoim de acordo com a tabela 3.

Tabela 3 - Produtividade e componentes de produção no primeiro cultivo de alface sobre diferentes resíduos, em diferentes alturas (cultivo 1 ).

PF - peso fresco; DCAB - diâmetro da cabeça; DCAU - diâmetro do caule; NF - número de folhas; $P C$ - ponto de colheita.

\begin{tabular}{lllll}
\hline Fatorial x testemunha & PF & DCAB & DCAU & NF \\
\hline & g planta $^{-1}$ & $(\mathrm{~cm})$ & $(\mathrm{cm})$ & \\
\hline Casca de Amen. 3 & $167,25^{*}$ & $26,08^{*}$ & 1,98 & $19,75^{*}$ \\
Casca de Amen. 6 & 101,58 & $24,08^{*}$ & 1,72 & 14,33 \\
Cana Triturada 3 & 24,96 & $13,75^{*}$ & $0,92^{*}$ & 7,62 \\
Cana Triturada 6 & 21,16 & $12,68^{*}$ & $0,81^{*}$ & 7,29 \\
T & 69,95 & 19,37 & 1,60 & 12,29 \\
\hline
\end{tabular}

* Difere da testemunha pelo teste de Dunnet a $5 \%$ de probabilidade. 


\begin{tabular}{|c|c|c|c|c|}
\hline Fatorial & PF & DCAB & DCAU & NF \\
\hline Resíduos & g planta $^{-1}$ & \multicolumn{2}{|c|}{$(\mathrm{cm})$} & \\
\hline Casca de Amen. & 134,41 a & $25,08 \mathrm{a}$ & $1,85 \mathrm{a}$ & $17,04 \mathrm{a}$ \\
\hline Cana Triturada & $23,06 \mathrm{~b}$ & $13,21 b$ & $0,87 b$ & $7,46 \mathrm{~b}$ \\
\hline \multicolumn{5}{|l|}{ Níveis } \\
\hline $3 \mathrm{~cm}$ & $96,10 \mathrm{a}$ & $19,91 \mathrm{a}$ & $1,45 \mathrm{a}$ & $13,68 \mathrm{a}$ \\
\hline $6 \mathrm{~cm}$ & $61,37 a$ & $18,38 a$ & $1,27 a$ & $10,81 \mathrm{a}$ \\
\hline
\end{tabular}

No segundo ciclo como se pode observar a partir da tabela 4 não houve diferença estatística entre os tratamentos para com a testemunha para as variáveis de crescimento PF, DCAB, DCAU, NF.

Tabela 4- Produtividade e componentes de produção no primeiro cultivo de alface sobre diferentes resíduos, em diferentes alturas (cultivo 2 ).

PF - peso fresco; DCAB - diâmetro da cabeça; DCAU - diâmetro do caule; NF - número de folhas; $\mathrm{PC}$ - ponto de colheita.

\begin{tabular}{|c|c|c|c|c|}
\hline $\begin{array}{c}\text { Fatorial } x \\
\text { testemunha }\end{array}$ & PF & DCAB & DCAU & NF \\
\hline & g planta $^{-1}$ & & & \\
\hline Casca de Amen. 3 & 156,83 & 25,29 & 1,58 & 23,66 \\
\hline Casca de Amen. 6 & 184,25 & 26,58 & 1,62 & 23,37 \\
\hline Cana Triturada 3 & 166,83 & 26,12 & 1,37 & 22,75 \\
\hline Cana Triturada 6 & 152,16 & 24,83 & 1,54 & 20,33 \\
\hline $\mathrm{T}$ & 127,29 & 24,82 & 1,52 & 19,87 \\
\hline \multicolumn{5}{|c|}{ * Difere da testemunha pelo teste de Dunnet a $5 \%$ de probabilidade. } \\
\hline Fatorial & PF & DCAB & DCAU & NF \\
\hline Resíduos & g planta $^{-1}$ & & & \\
\hline Casca de Amen. & $170,54 \mathrm{a}$ & $25,94 \mathrm{a}$ & $1,60 \mathrm{a}$ & $23,52 \mathrm{a}$ \\
\hline Cana Triturada & 159,50 a & $25,48 \mathrm{a}$ & $1,45 \mathrm{a}$ & $21,54 \mathrm{a}$ \\
\hline \multicolumn{5}{|l|}{ Níveis } \\
\hline $3 \mathrm{~cm}$ & 161,83 a & $25,71 \mathrm{a}$ & $1,48 \mathrm{a}$ & $23,20 \mathrm{a}$ \\
\hline $6 \mathrm{~cm}$ & $168,21 \mathrm{a}$ & $25,70 \mathrm{a}$ & $1,58 \mathrm{a}$ & $21,85 \mathrm{a}$ \\
\hline
\end{tabular}

Letras iguais nas colunas não diferem significativamente pelo teste de Tukey a $5 \%$ de probabilidade.

Em relação ao carbono da biomassa microbiana apresentados na Tabela 5, observa-se que a cobertura de ambos os níveis da casca de amendoim foram melhores estatisticamente do que a cana triturada. A atividade da enzima desidrogenase e o Coeficiente metabólico $\left(q \mathrm{CO}_{2}\right)$ obteve semelhança entre os tratamentos (Tabela 5). 
Tabela 5 - Carbono da biomassa microbiana do solo e atividade da enzima desidrogenase e respiração basal do solo no cultivo da alface, sobre diferentes resíduos, em diferentes alturas, avaliados no final do segundo cultivo.

\begin{tabular}{|c|c|c|c|c|}
\hline $\begin{array}{l}\text { Fatorial } x \\
\text { testemunha }\end{array}$ & CBMS & Desidrogenase & Respiração & $\mathrm{QCO}_{2}$ \\
\hline & $\mu g^{-1}$ & $\begin{array}{l}\text { mg TTF g }{ }^{-1} \text { solo }^{-1} \\
24 \mathrm{~h}^{-1}\end{array}$ & $\begin{array}{l}\text { mg de } \mathrm{C}-\mathrm{CO}_{2} \mathrm{~kg}^{-1} \\
\text { solo dia }\end{array}$ & $\begin{array}{c}\mathrm{mgC}-\mathrm{CO}_{2} \mathrm{~g}^{-1} \mathrm{BMS}-\mathrm{C} \\
\mathrm{dia}^{-1} \\
\end{array}$ \\
\hline Casca de Amen. 3 & 34,38 & 3,83 & 5,52 & 0,20 \\
\hline Casca de Amen. 6 & 47,72 & 2,68 & 8,02 & 0,17 \\
\hline Cana Triturada 3 & 29,24 & 2,72 & 6,42 & 0,22 \\
\hline Cana Triturada 6 & 29,10 & 1,95 & 5,17 & 0,18 \\
\hline $\mathrm{T}$ & 32,58 & 2,86 & 6,88 & 0,21 \\
\hline
\end{tabular}

* Difere da testemunha pelo teste de Dunnet a $5 \%$ de probabilidade.

\begin{tabular}{|c|c|c|c|c|}
\hline Fatorial & CBMS & Desidrogenase & Respiração & $\mathrm{QCO}_{2}$ \\
\hline Manejos & $\mu g^{-1}$ & $\begin{array}{c}\text { mg TTF g }{ }^{-1} \text { solo }^{-1} \\
24 \mathrm{~h}^{-1}\end{array}$ & $\begin{array}{l}\text { mg de } \mathrm{C}-\mathrm{CO}_{2} \mathrm{~kg}^{-1} \\
\text { solo dia }\end{array}$ & $\begin{array}{c}\mathrm{mgC}-\mathrm{CO}_{2} \mathrm{~g}^{-1} \mathrm{BMS}-\mathrm{C} \\
\mathrm{dia}^{-1}\end{array}$ \\
\hline Casca de Amen. & $41,05 \mathrm{a}$ & 3,25 & $6,77 \mathrm{a}$ & $0,18 \mathrm{a}$ \\
\hline Cana Triturada & $29,17 b$ & 2,33 & $5,80 \mathrm{a}$ & $0,20 \mathrm{a}$ \\
\hline \multicolumn{5}{|l|}{ Níveis } \\
\hline $3 \mathrm{~cm}$ & 31,81 a & $3,28 \mathrm{a}$ & 5,97 a & 0,21 a \\
\hline $6 \mathrm{~cm}$ & $38,41 \mathrm{a}$ & 2,31 a & $6,60 \mathrm{a}$ & $0,18 \mathrm{a}$ \\
\hline
\end{tabular}

Letras iguais nas colunas não diferem significativamente pelo teste de Tukey a $5 \%$ de probabilidade.

\section{DISCUSSÃO}

De acordo com Oliveira et al. (2008) em estudo sobre coberturas mortas em cultura de alface sob manejo orgânico, obteve avaliações efetuadas ao final do primeiro ciclo de cultivo da hortaliça indicaram que todas as coberturas mortas foram eficientes no controle da vegetação espontânea reinfestante e que em ambos os ciclos de cultivo da alface, foram constatados valores mais elevados de massa fresca e diâmetro da parte aérea colhida (cabeça) com as coberturas mortas de leguminosas. Ele ainda ressalta que os benefícios trazidos pelas coberturas mortas de leguminosas resultaram, principalmente, da disponibilização de nitrogênio para a cultura, liberado através da acelerada decomposição dos resíduos, onde a cultura irá obter um melhor desenvolvimento e consequentemente um maior peso de massa fresca.

A palhada de leguminosas, depositada de forma fragmentada na superfície do solo, apresenta rápida decomposição e liberação de nutrientes, o que favorece o desempenho das culturas. Por outro lado, as gramíneas normalmente apresentam decomposição mais lenta, podendo inclusive acarretar imobilização de nutrientes no solo (ESPINDOLA et al., 2006), onde causará uma deficiência desse nutriente, assim prejudicando o desenvolvimento da planta. Castro (2007) observou em um trabalho realizado com cobertura vegetal e indicadores microbiológicos 
que não houve diferença significativa entre os tratamentos para os indicadores biológicos analisados e isso ocorreu provavelmente em razão da necessidade de um tempo maior para estabelecimento da vegetação, permitindo maior cobertura e sua expressão sobre a microbiota.

Anderson; Domsch (1985); Jakelaitis et al., (2007) o qual prediz que, à medida que a biomassa microbiana se torna eficiente em utilizar os recursos, menor quantidade de carbono é perdida como $\mathrm{CO}_{2}$ pela respiração, sendo este imobilizado no tecido microbiano; consequentemente, menor $\mathrm{qCO}_{2}$ representa biomassa microbiana mais estável, ou ambiente com menor grau de distúrbio. $\mathrm{O} \mathrm{qCO}_{2}$ apresenta ao solo uma maior estabilidade entre os manejos realizados.

\section{CONCLUSÃO}

Conclui-se que a utilização da cobertura morta trouxe resultados benéficos ao solo, como à redução de ervas espontânea, o uso da casca de amendoim melhorou a biomassa microbiana do solo e aumentou a produtividade da alface nos dois cultivos. E apenas no primeiro cultivo houve diferenças quanto a altura da camada de resíduos, sobressaindo-se a altura de $3 \mathrm{~cm}$ do amendoim como a de melhor produção de alface.

\section{REFÊRENCIAS}

ANDRADE JÚNIOR, V. C. de et al. Emprego de tipos de cobertura de canteiro no cultivo da alface. Hortic. Bras. Brasília, v.23, n.4, p.899-903, out-dez., 2005. Disponível em: < http://www.scielo.br/pdf/hb/v23n4/a07v23n4.pdf >. Acesso em: 21 abr. 2016.

BATISTA, M. A. V. et al. Atributos microbiológicos do solo e produtividade de rabanete influenciados pelo uso de espécies espontâneas. Hortic. Bras. Alagoinha, v.31, n.4, out-dez., 2013. Disponível em: < http://www.scielo.br/pdf/hb/v31n4/13.pdf >. Acessa em: 21 abr. 2016.

CAMARGO FILHO, W. P. de. Perfil da olericultura no Estado de São Paulo. São Paulo.2013. Disponível em: < http://www.bbagro.com.br/artigos/Perfil\%20da\%200lericultura\%20SP.pdf>. Acesso em: 21 abr. 2016.

CASTRO, P.T.C. Cobertura Vegetal e Indicadores Microbiológicos de Solo em Talude Revegetado. Viçosa- MG, $2007 . \quad$ Disponível em: http://locus.ufv.br/bitstream/handle/123456789/4480/texto\%20completo.pdf?sequence=1\&isAll owed $=y>$. Acesso em 23 jun. 2017.

JAKELAITIS, A.; SANTOS, J.B.; VIVIAN, R.; SILVA, A.A. Atividade Microbiana e Produção de Milho (Zea mays) e de Brachiaria brizantha sob Diferentes Métodos de Controle de Plantas Daninhas. Planta Daninha, Viçosa-MG, v. 25, n. 1, p. 71-78, Mar., 2007. Disponível em:< http://www.scielo.br/pdf/pd/v25n1/a08v25n1.pdf>. Acesso em: 28 jun. 2017.

OLIVEIRA, F. F. de et al. Avaliação de coberturas mortas em cultura de alface sob manejo orgânico. Hortic. Bras. Seropédica - RJ, v.26, n.2, abr-jun., 2008. Disponível em: < http://www.scielo.br/pdf/hb/v26n2/17.pdf >. Acesso em: 21 abr. 2016.

RESENDE, F. V. et al. Uso de cobertura morta vegetal no controle da umidade e temperatura do solo, na incidência de plantas invasoras e na produção da senhora em cultivo de verão. Ciênc. Agrotec, Lavras, v.29, n.1, p.100-105, jan-fev., 2005. Disponível em: < http://www.alice.cnptia.embrapa.br/bitstream/doc/778369/1/resendeuso.pdf $>$. Acesso em: 21 abr. 2016. 
VALARINI, P. J. et al. Qualidade do solo em sistema de produção de hortaliças orgânicas e convencional. Hortc. Bras. Ibuína e Socorro - SP, v.29, n.4, out-dez., 2011. Disponível em: < http://www.scielo.br/pdf/hb/v29n4/a07v29n4.pdf >. Acesso em: 21 abr. 2016. 